\title{
Ambiente e saúde mental: experiência clínica com estudantes de uma universidade pública.
}

\author{
Environment and Mental Health: Clinical Experience with \\ Students from a Public University
}

Ambiente y salud mental: experiencia clínica con estudiantes de una universidad pública.

Georgiano Joaquim Pereira Antonio dos Santos*, Ana Carlota Pinto Teixeira**, Maria Auxiliadora Motta Barreto ${ }^{* * *_{3}}$

\begin{abstract}
RESUMO
O estudo da saúde mental de universitários vem sendo pauta de discussões há alguns anos no Brasil e, recentemente, essa questão ganhou maior repercussão devido aos casos de adoecimento nas universidades. Este estudo teve como objetivo demostrar a importância da implementação de programas de atenção à saúde mental no contexto universitário, a partir da experiência de estágio clinico com estudantes de uma universidade pública, como forma de promoção de saúde. Entre março e setembro de 2018 foram atendidas 21 pessoas (201 sessões), com prevalência do gênero feminino (71,43\%). Além disso, 47,62\% das queixas trazidas correspondem a transtornos psiquiátricos ou a sensações que remetem a esses transtornos, enquanto $71,43 \%$ dos
\end{abstract} Palavras-chaves: Adoecimento Psíquico. Saúde Mental e Estudantes Universitários. Serviços de Saúde Mental na Universidade. Prevenção Psicológica.

\footnotetext{
* Psicólogo, Mestrando em Psicologia Escolar e Desenvolvimento Humano pelo Instituto de Psicologia da Universidade de São Paulo (IPUSP). Graduado em Psicologia pelo Centro Universitário Salesiano de São Paulo (UNISAL). ORCID: https://orcid.org/00000002-7043-3624. Contacto: georgiano@usp.br

** Psicólogo, Mestre em Psicologia da Saúde pela Universidade Metodista de São Paulo. Graduada em Psicologia pela Faculdade Salesiana de Filosofia, Ciências e Letras. Contacto: anacarlotapsi@yahoo.com.br *** Psicóloga, Doutora em Psicologia como Profissão e Ciência pela Pontifícia Universidade Católica de Campinas (PUC). Mestre em Educação pelo Centro Universitário Salesiano de São Paulo (UNISAL). Graduada em Psicologia pela Faculdade Salesiana de Filosofia, Ciências e Letras. Docente da Universidade de São Paulo (USP). ORCID: https:// orcid.org/0000-0002-3149-0482. Contacto: maribarreto@usp.br
} 
participantes totais relatam ter alguma dificuldade acadêmica ou sentirem-se desmotivados em relação ao curso. Os dados aqui expostos oferecem subsídios para a continuação e ampliação do serviço e o reconhecimento de sua utilidade para a comunidade acadêmica.

\begin{abstract}
The study of mental health among college students has been the subject of debate for the past few years in Brazil and has recently gained popularity due to cases of mental illness at universities. This study was aimed at demonstrating the importance of implementing mental healthcare programs in the university context, based on the experience of a clinical internship with students at a public university, as a way to promote health. Between March and September 2018, 21 people were treated (201 sessions), most of which were female (71.43\%). Additionally, $47.62 \%$ of the complaints addressed refer to psychiatric disorders or feelings that are tied to these disorders, while $71.43 \%$ of all participants claim to have academic difficulties or feel unmotivated in relation to their studies. The data presented here offer input for continuing and expanding the service and the recognition of its usefulness to the academic community.
\end{abstract}

\section{RESUMEN}

El estudio de la salud mental de los estudiantes universitarios es objeto de discusión desde hace algunos años en Brasil y, recientemente, ha ganado mayor repercusión debido a los casos de enfermedad en las universidades. Este estudio busca demostrar la importancia de implementar programas de atención a la salud mental en el contexto universitario, a partir de la experiencia de las prácticas clínicas con estudiantes de una universidad pública, como forma de promover la salud. Entre marzo y septiembre de 2018 se han atendido a 21 personas (201 sesiones), con predominio del género femenino (71,43\%). Además, 47,62\% de las quejas aportadas corresponden a trastornos psiquiátricos o a sensaciones que remiten a estos trastornos, mientras que $71,43 \%$ del total de los participantes manifestó tener alguna dificultad académica o sentirse desmotivado con relación a su carrera. Los datos aquí expuestos ofrecen ayudas para la continuación y ampliación del servicio y el reconocimiento de su utilidad para la comunidad académica.
Keywords: Mental illness. Mental health and college students. Mental health services at universities. Psychology prevention.

Palabras clave: Enfermedad mental. Salud mentaly estudiantes universitarios. Servicios de salud mental en la universidad. Prevención psicológica. 


\section{Introdução}

A saúde mental de estudantes universitários começou a ser discutida nos Estados Unidos e na Europa a partir da década de 1960 e, no contexto brasileiro, podemos visualizar os primeiros estudos referentes a essa população em 1958 (Cerchiari, Caetano \& Faccenda, 2005; Venturini \& Goulart, 2016). Atualmente, vemos que a discussão sobre esse tema voltou a se tornar pauta nos ambientes acadêmicos e na mídia nacional devido ao aumento da incidência de adoecimento psíquico em universitários.

Para se ter uma ideia da gravidade do problema, de acordo com a V Pesquisa Nacional de Perfil Socioeconômico e Cultural dos (as) Graduandos (as) das IFES (2018), em um universo de 424 mil estudantes pesquisados, 83,3 \% apresentaram alguma dificuldade emocional, enquanto que $63,6 \%$ relataram problemas ou sensações relacionadas a ansiedade.

A entrada na universidade aproxima os estudantes das exigências da sociedade no que se refere à atuação profissional e cidadã, exigindo eficácia e adaptação às novas situações, com pressão e aceitação externas. Torna-se, então, importante que o estudante universitário aprenda a desempenhar seu ofício de estudante (Coulon, 2017, p. 1243), isto é, "descobrir as rotinas, as evidências, as regras, os novos códigos da universidade", os quais são totalmente diferentes se comparados as experiências anteriores, como por exemplo o ensino médio.

Nesse estudo, a compreensão da saúde de estudantes universitários assume uma perspectiva multidimensional, em consonância com o que é postulado pela Organização Mundial de Saúde (1948), e com as reflexões de Accorsi (2015), que nos mostra que as possíveis fragilidades que os estudantes trazem consigo, aliadas aos desafios do ambiente universitário, podem potencializar, ou mesmo causar, quadros de adoecimento físico ou mental.

De acordo com Andrade et al (2016) e Venturini e Goulart (2016) a implementação de serviços de psicologia com enfoque no atendimento a estudantes universitário pode ser utilizada como uma das ferramentas que as universidades podem dispor para possibilitar uma trajetória acadêmica menos difícil para seus discentes, agindo como potencializadora de saúde. 
Entretanto, o que se nota é um grande distanciamento entre discurso e prática, isto é, a efetivação de estratégias dentro das universidades para a promoção de saúde mental dos seus discentes não acontece de forma consistente e generalizada. Nesse contexto, observamos a existência de ações isoladas de algumas universidades em oferecer algum tipo de programa ou serviço de atenção à saúde mental, ao mesmo tempo em que cresce a demanda de suportes por parte da população universitária para atender suas dificuldades (Accorsi, 2015).

Nesse sentido, este estudo tem como objetivo demonstrar a importância da implementação de programas de atenção à saúde mental no contexto universitário, a partir do relato de experiência de um estágio clínico com estudantes de uma universidade pública, como forma de promoção de saúde.

\section{Universidade como - possivel - 'ambiente facilitador'}

Nesse estudo, a compreensão da saúde de estudantes universitários assume uma perspectiva multidimensional, em consonância com o que é postulado pela OMS (1948), e com as reflexões de Accorsi (2015), que nos mostra que as possíveis fragilidades que os estudantes trazem consigo, aliadas aos desafios do ambiente universitário, podem potencializar, ou mesmo causar, quadros de adoecimento físico ou mental.

Assim, focalizando no ambiente universitário e, mais especificamente, na instituição universitária, propomos uma reflexão sobre o fato de que esta pode, sim, se tornar um ambiente facilitador de saúde para os seus discentes. Para isso, faremos uma breve exposição de algumas ideias de Winnicott. A utilização dessa perspectiva se mostra útil pois se evidenciam características peculiares do ambiente institucional que podem interferir na promoção de saúde dos estudantes universitários.

Para isso, primeiramente, é importante compreender como se dá o encontro inicial do indivíduo com o mundo e sua busca por um ambiente que o permita continuar sendo. Conforme aponta Mizrahi (2008, p. 63) esse encontro inicial, na teoria winnicottiana, ocorre a partir "da compatibilidade potencial entre suas necessidades primeiras e o mundo tal como este se apresenta no manejo sensível da mãe ou de seu substituto". Esse "se apresentar" se relaciona com o grau de adaptabilidade da mãe ambiente "às necessidades singulares de seu filho, 
sendo por ele sentida como parte indiferenciada de seu self" (ibidem). Winnicott utiliza esse argumento para demostrar a diferença entre ser e reagir, entre "a vida podendo fluir como continuidade e encontrando o ambiente adaptado e aquela outra que, de modo distinto, emerge respondendo às intrusões perturbadoras" (Mizrahi, 2008, p. 63).

Surge a pergunta: de que maneira será feito o contato? Como parte do processo vital do indivíduo, ou como consequência da intranquilidade do ambiente? Digamos que a adaptação ativa seja quase perfeita. [...] O movimento do próprio indivíduo [...] descobre o ambiente. Isto, repetido se transforma num padrão de relacionamento. Num caso menos feliz, o padrão de relacionamento se baseia no movimento do ambiente. Isto merece o nome de intrusão que é imprevisível por não ter relação alguma com o processo vital do próprio indivíduo (Winnicott, 1990, pp. 148-149).

Winnicott nos mostra que o modo como o ambiente se adapta às necessidades do indivíduo terá grande influência na vivência de uma "vitalidade [...] que encontra espaço para fluir" e outra que "precisa lidar basicamente com situações adversar" (Mizrahi, 2008, p. 63). Esse quadro não se restringe aos primeiros anos de vida da criança, podendo ser visualizado também em momentos mais tardios do desenvolvimento individual, com a diferença de que a "adaptação ambiental torna-se, em certo sentido, menor e a objetividade do mundo se faz mais presente" (ibidem). Nesse sentido, a ideia geral de "ambiente" presente em Winnicott diz respeito "ás condições físicas e psicológicas necessárias para o amadurecimento emocional do ser humano" (Araújo, 2011, p. 213).

A partir dessa ótica, podemos compreender o social e, por conseguinte, a universidade, objeto desse estudo, como uma extensão desse ambiente inicial, o qual terá que, necessariamente, oferecer dentro de suas estruturas, subsídios básicos para o desenvolvimento de indivíduos saudáveis, isto é, qualidades ambientais favoráveis e suficientes às necessidades destes. Para Winnicott, essas condições receberão o nome de ambiente suficientemente bom e permitirão o desenvolvimento global do indivíduo.

Há genes que determinam padrões e tendências herdadas para o crescimento e a aquisição de maturidade; não há crescimento emocional, no entanto, a não ser em relação à provisão ambiental, que precisa 
ser satisfatória. Pode-se notar que a palavra "perfeito" não entra nessa frase. A perfeição pertence às máquinas, e as imperfeições próprias da adaptação humana às necessidades constituem uma característica essencial do meio ambiente facilitador (Winnicott, 1968/1999, p. 148).

Entretanto, essa "condição satisfatória" só se torna possível, nesse momento inicial do desenvolvimento, caso o bebê possa experimentar o cuidado que recebe como uma criação proveniente de si, ou seja, o manejo ambiental satisfatório poderá ser experienciado como algo que o constitui (Mizrahi, 2008). Winnicott dará o nome de objeto subjetivo a esse paradoxo, no qual "a criação pessoal só pode ser sentida como tal quando sustentada pelo cuidado real "externo" que o indivíduo recebe efetivamente do mundo" (Mizrahi, 2008 p. 65). A mãe, ao proporcionar essa vivência, permite que o lactante possa vivenciar a ilusão de que a realidade apresentada foi criada em detrimento a sua necessidade (Winnicott, 1990). Permitir essa ilusão será essencial para "o estabelecimento inicial da capacidade de manter relações com o mundo real" (Mizrahi, 2008, p. 65), o que só se torna possível no encontro "satisfatório entre o indivíduo e o ambiente" (ibidem).

Dessa maneira, o encontro satisfatório entre indivíduo e ambiente universitário, se torna essencial para que a universidade seja experienciada pelos seus discentes como um ambiente facilitador de desenvolvimento pessoal e relacional, não apenas em sentido concreto, como também em sentido simbólico. Entretanto, em muitos casos, o que se nota nos contextos institucionais é um desencontro entre indivíduo e instituição: esta falha em oferecer subsídios essenciais e satisfatórios para a promoção do desenvolvimento dos discentes, ao passo que estes possuem déficits desenvolvimentais provenientes de suas próprias histórias de vida, isto é, déficits anteriores à entrada no ensino superior, que acabam por não ser reparados minimamente por essas instituições devido à falta de oferta de subsídios básicos para tal.

Ao falarmos de subsídios básicos oferecidos pelas universidades, retomamos a ideia de Winnicott (1968/1999) de ambiente satisfatório, mas não perfeito, que proporcione boas relações interpessoais e, nesse caso, serviços, programas e estruturas satisfatórias aos discentes como forma de cuidado. Podemos citar, como exemplo, os serviços de orientação e de saúde integral, em especial os relacionados a saúde mental, ofertados por algumas universidades, os projetos sociais, de esportes e 
lazer, as oportunidades acadêmicas e a possibilidade de vínculos com colegas de curso e professores.

A oferta dos serviços citados, e, principalmente, a vinculação entre os estudantes, bem como entre corpo discente e docente, caracterizam-se como pontos cruciais para que a experiência universitária possa ser vivida com 'experiência', pois para isso é necessário a existência de um outro sentido como real. Apenas esse tipo de experiência permite o desenvolvimento saudável do ser humano.

\section{Metodologia}

Este estudo trata-se de um relato de experiência referente a acompanhamentos clínicos realizados em um serviço de atendimento psicológico ao aluno de uma universidade pública do interior do estado de São Paulo. Os dados referem-se a atendimentos realizados com 21 estudantes, entre março e setembro de 2018, num total de 201 sessões. As vinhetas clínicas apresentadas foram colhidas ao longo dos atendimentos.

A implementação do serviço nessa universidade ocorreu em 2016, por iniciativa de uma docente da $^{1}$ instituição em questão. $\mathrm{O}$ serviço possui parceria com o curso de psicologia do Centro Universitário Salesiano de São Paulo (UNISAL), Campus São Joaquim, e, desde sua criação, recebe um estagiário do último ano deste curso que se torna responsável por realizar o acompanhamento e orientação dos alunos que procuram o serviço.

\section{Caracterização e estruturação do serviço}

A modalidade de atendimento utilizada foi, predominantemente, a de psicoterapia breve individual de cunho psicanalítico, com alguns atendimentos pontuais de aconselhamento e orientação. Todos os casos eram supervisionados por uma professora psicóloga do UNISAL com prática na área clínica, tendo como base os relatos de sessão.

O serviço contava com uma sala para atendimento individual, com funcionamento de segunda-feira a sexta-feira, em pelo menos um período do dia (manhã ou tarde) e sua procura poderia ser feita presen-

1 Esta docente também possui formação em psicologia 
cialmente ou via e-mail e/ou telefone institucional. O estagiário ficava responsável por cumprir 20 horas semanais na instituição podendo atender, no máximo, três alunos por período. A divulgação do serviço ficava a cargo da instituição e sua utilização era aberta a todos os alunos, Graduação e Pós-Graduação, dando-se preferência aos primeiros.

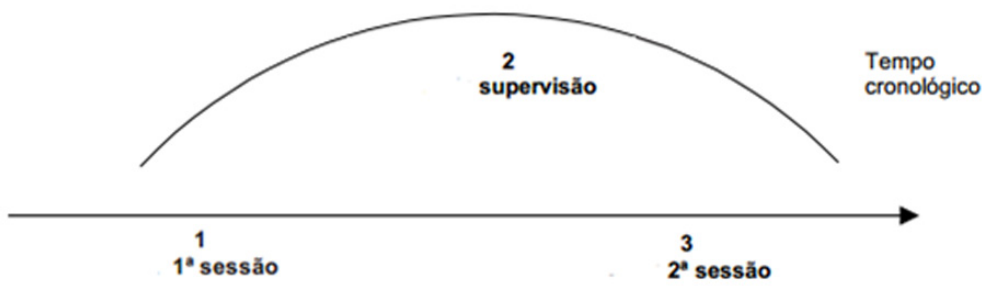

Figura 1: Três tempos lógicos para a definição do atendimento psicoterapêutico

Fonte: Peres, Santos \& Coelho (2003) - adaptado

Após uma maior compreensão dos casos, geralmente em no mínimo 12 sessões, realizava-se uma avaliação individual de cada paciente que poderiam suceder três ações: 1) encaminhamento para outros profissionais (psicólogos e psiquiatras, em sua maioria); 2) desligamento do serviço (com ou sem indicação de continuação do processo psicoterapêutico com outro profissional) ou; 3) continuação do acompanhamento na instituição. Nos três casos, os critérios utilizados foram: a) nível de sofrimento psíquico (gravidade do sintoma) e; b) situação socioeconômica. A adoção desses procedimentos ocorreu devido a modalidade de atendimento adotada (psicoterapia breve) e a alta demanda de procura pelo serviço, o que, consequentemente, não possibilitava a oferta de atendimento prolongado aos alunos, salvo exceções ${ }^{2}$.

\section{Resultados}

Entre os meses de março e setembro de 2018 foram atendidas 21 pessoas, num total de 201 sessões. A disponibilidade de horário de um

2 Em alguns casos, devido ao alto nível de sofrimento psíquico (gravidade do sintoma) e o baixo poder aquisitivo do aluno, optou-se pelo prolongamento do atendimento. 
único profissional responsável por essa atividade determinou a abrangência de atendimentos. A maioria dos estudantes que procuraram o serviço encontram-se na faixa de 20 a 23 anos $(66,67 \%)$. No que se refere ao sexo, 71,43\% dos pacientes são do sexo feminino, o que mostra uma maior procura de mulheres pelo serviço de psicologia na instituição.

No que se refere a distribuição por nível de formação, $76,19 \%$ dos usuários do serviço são graduandos, 9,52\% são mestrandos e 14,29\% são doutorandos. Em relação à graduação, a procura pelo serviço, distribuído por curso, se deu da seguinte forma: Engenharia Química (50\%), Engenharia Ambiental (31,25\%), Engenharia Bioquímica (12,50\%) e Engenharia de Produção (6,25\%).

Quanto às queixas dos usuários, apenas 22,73\% iniciaram a psicoterapia breve com algum diagnóstico já estabelecido, os quais foram: Transtorno de ansiedade generalizado (CID 10 - F411), Transtorno de Ansiedade não especificado (CID 10 - F419), Transtorno de pânico (CID 10 - F41.0), Transtorno misto ansioso e depressivo (CID 10 F412). Dentre esses pacientes, $20 \%$ iniciaram tratamento psiquiátrico, mas não deram continuidade, enquanto que $80 \%$ realizava acompanhamento psiquiátrico em conjunto a psicoterapia.

Os usuários relataram que os transtornos psiquiátricos apareceram durante sua formação superior - nessa instituição ou em anteriores - e que estão ligados a eventos estressores de ordem acadêmica (ex.: repetir uma matéria), de perda (ex.: falecimento de pessoas próximas) e/ou relacional (ex.: problemas nos relacionamentos familiares). Relataram, ainda, o comprometimento do desempenho acadêmico e/ou de suas relações dentro e fora da universidade devido aos sintomas.

“[...] tomar decisões hoje em dia é muito difícil, fico indecisa no que escolher [...] antes não era assim. A síndrome do pânico não atrapalha apenas na faculdade, mas na minha vida como um todo" (Paciente B, 22 anos).

"Estou indo muito mal nas matérias, ando muito estressada, por isso me afasto de todos. Me sinto muito ansiosa, tenho apertos no peito [...] não estou conseguindo me relacionar com as pessoas e dar conta da faculdade. [...] já pensei em trancar a faculdade" (Paciente $\mathrm{H}, 28$ anos). 
Sobre a utilização de medicamentos, os pacientes relatavam medo devido a possível dependência e/ou baixa eficácia destes no que se refere ao "sentir-se mal".

"Procurei ajuda porquê não quero voltar a tomar remédio, ele tirava as minhas expressões, não conseguia reagir as situações". (Paciente A, 29 anos).

"Tomo remédio por causa da síndrome de pânico, mas ele só resolve os sintomas físicos. O mal-estar interno continua forte e me sufoca" (Paciente B, 22 anos).

"Sinto que o remédio tira a ansiedade, mas não o resto" (Paciente C, 22 anos).

Observou-se ainda que $47,62 \%$ das queixas trazidas correspondem a transtornos psiquiátricos ou a sensações que remetem a esses transtornos. A ansiedade aparece como a mais presente dentro desse grupo (80\%), seguida pela sensação de estar deprimido (10\%) e distúrbios alimentares (10\%). É necessário ressaltar que muitos pacientes apresentam comorbidade das sensações citadas, entretanto, optamos por expor os quadros que se mostraram mais presente ao longo do acompanhamento.

“[...] estou muito ansiosa, acho que é porque ando fazendo muitas coisas" (Paciente D, 20 anos).

"[...] sou muito ansiosa e nervosa. As vezes tenho falta de ar devido a isso, a respiração fica difícil" (Paciente G, 22 anos).

Por outro lado, 52,38\% dos usuários apresentaram dificuldades psicológicas não psiquiátricas relativas a autoconhecimento, dificuldades nos relacionamentos interpessoais, baixa autoestima, desânimo, timidez e dificuldades de aprendizagem, desempenho insatisfatório e/ou insatisfação com o curso escolhido.

No que se refere ao ambiente universitário, haviam queixas referentes as dificuldades em se relacionar com colegas e professores, bem como sobre a adaptação no curso e na cidade. Nesses casos, o ambiente universitário era vivenciado como lugar de solidão, hostil, de não pertencimento:

"Sinto falta de me relacionar com proximidade com as pessoas. Não tenho ninguém próximo aqui. Me sinto muito sozinha principal- 
mente no final de semana, quando todo mundo vai embora. Não gosto da cidade, do curso que escolhi, das pessoas daqui. $\mathrm{O}$ que mais sinto falta aqui é de me relacionar com os outros. No laboratório que fico sou a única mulher. Quando fiz minha graduação não era assim. Sinto falta de ter amigos pra conversar" (Paciente E, 25 anos).

"A faculdade não me ajuda em nada, sempre parece que quer me afundar ainda mais. Já cansei de ouvir de professores e até da coordenadora do curso que eu devia desistir disso" (Paciente B, 22 anos).

"Não gosto de ficar sozinho aqui, fico depressivo com isso. Férias e feriados são muito difíceis pra mim [...]" (Paciente G, 22 anos).

"Não gosto da cidade e nem das pessoas daqui. A maioria das relações que são construídas são devido a interesse, não sinto que são relações verdadeiras. É diferente na minha cidade." (Paciente H, 28 anos).

Sobre a vivência acadêmica, é necessário ressaltar que $71,43 \%$ dos participantes totais relatam ter alguma dificuldade ou sentirem-se desmotivados em relação ao curso. A importância de um acompanhamento educacional se faz presente nesses casos para proporcionar uma reflexão sobre a trajetória acadêmica e possíveis transformações.

“[...] as vezes me sinto muito incapaz em realizar qualquer coisa. Questiono até mesmo o doutorado, as vezes acho que não deveria estar aqui" (Paciente D, 30 anos).

"Não queria fazer mestrado, mas como não arrumei nenhum emprego e não queria voltar para a casa dos meus pais, acabei prestando a prova e passei" (Paciente E, 25 anos).

"Apesar das pessoas me acharem boa e capaz, não sinto isso. Tenho medo que descubram que não sou tudo isso" (Paciente F, 23 anos).

\section{Discussão e conclusões}

Como podemos notar nesse relato de experiência, a maior procura pelo serviço de psicologia foi realizada predominantemente por pessoas do sexo feminino. Esse dado encontra-se em consonância com os estudos de Cerchiari et al. (2005), Neves e Dalgalarrondo (2007) e Sou- 
za et al (2013) os quais demonstraram que o sexo feminino utiliza mais os serviços de saúde mental, apresenta maiores queixas de sofrimento mental e maiores dificuldades psicossociais.

Todavia, apesar da maior demanda de serviços estar relacionada ao sexo feminino, observa-se nas universidades de engenharia, como no caso desse estudo, uma menor participação de mulheres. Um estudo realizado por Nascimento (2017), na Universidade Federal da Bahia (UFBA), aponta que entre os alunos ingressantes na universidade em 2013, cerca de 32,2\% eram mulheres. Esse dado encontra ressonância no censo da educação superior realizado pelo Instituto Nacional de Estudos e Pesquisas Educacionais (INEP, 2015), no qual as mulheres representam 31,5\% dos matriculados nas áreas de engenharia, produção e construção.

A maior procura pelo serviço de psicologia por mulheres pode se relacionar aos papeis de gênero existentes em nossa sociedade, isto é, aos modelos de masculinidade e feminilidade que aproximam mulheres das preocupações com o autocuidado e, consequentemente, da busca pelos serviços de saúde e afastam os homens dessas mesmas questões (Gomes et al, 2011). Esses papéis de gênero são compostos por valores culturais e históricos que relacionam certas características como relatar as próprias fragilidades físicas e emocionais como sendo apenas femininas (Costa-Junior \& Maia, 2009).

Em relação ao adoecimento de universitários, conforme aponta Accorsi (2015) e Rios et al (2019), é possível constatar maior prevalência de problemas de saúde mental na população universitária se comparado com a população da mesma faixa etária que não se encontra na universidade. Porém, devemos tomar cuidado em não culpabilizar um dos polos dessa trama, ou seja, universitários e universidade, mas sim compreender que a individualidade, coletividade e o ambiente que acolhe essas instâncias, caminham juntos e em inter-relação e que disso surge uma experiência rica e diversa na qual o adoecimento pode fazer parte (Accorsi, 2015).

A entrada na Universidade é perpassada por transformações significativas no modo de agir e pensar do universitário e esse processo pode ser perpassado por idealizações, ansiedades, conflitos e angústias (Castro, 2017). Além disso, esse novo contexto traz exigências so- 
ciais de maior autonomia por parte do aluno, o que pode causar estresse, evasão e outras dificuldades entre aqueles que ainda não estão preparados para essa nova fase da vida (Dias et al, 2019).

Entretanto, conforme se verifica nas falas dos universitários, podemos constatar que o adoecimento no contexto acadêmico não se relaciona apenas às instituições universitárias, mas também a questões externas, bem como pela insuficiência de recursos internos dos indivíduos para lidar com as situações adversas da vida (Accorsi, 2015). Esses aspectos, em conjunto com as exigências acadêmicas podem causar estresse, problemas orgânicos, dificuldades de relacionamento, baixa produtividade escolar, angústias, estados de depressão, apatia e, em casos mais graves, a perda do interesse pela vida, que pode suscitar no suicídio.

Os atendimentos utilizados como base para esse estudo deixam claro que, apesar da grande maioria das dificuldades relatadas manifestarem-se após o início da formação acadêmica, existem características peculiares da trajetória de vida de cada indivíduo (traumas, distancia da família, dificuldades financeiras, dentre outras), bem como a falta de mecanismos internos adequados para lidar com essas situações, fatores que contribuem para o adoecimento do estudante universitário, o que vai ao encontro à pesquisa de Accorsi (2015).. Apesar disso, é de responsabilidade das instituições universitárias oferecerem subsídios satisfatório, mas não perfeitos, para a promoção de saúde de seus estudantes.

A oferta de subsídios satisfatórios dentro das universidades para dar respaldo a essas problemáticas torna-se essencial, pois como aponta Winnicott (1968/1999, p. 148) “não há crescimento emocional, no entanto, a não ser em relação à provisão ambiental, que precisa ser satisfatória". Todavia, não podemos esquecer que as instituições ao serem promotoras de um ambiente facilitador aos seus discentes também estão se "alimentando" disso, ou seja, a saúde institucional está diretamente ligada a saúde individual dos integrantes dessa instituição, e vice-versa, pois, como postula Winnicott (1967/1999, p. 3) “a saúde social depende da saúde individual".

Pode-se concluir, à face do que foi exposto neste relato de experiência, que o estudante universitário se depara, ao longo de sua formação, 
com inúmeras situações de risco que podem interferir em sua saúde mental e, por conseguinte, em sua qualidade de vida. Entretanto, é impossível afirmar que as dificuldades psicológicas e psiquiátricas enfrentadas por esses indivíduos possuem relação direta apenas com o ambiente institucional das universidades.

Ressalta-se que, devido as limitações do serviço, os dados aqui expostos não refletem o universo de discentes que necessitam de alguma ajuda ou tratamento. Contudo, oferecem subsídios para a continuação e ampliação do serviço e o reconhecimento de sua utilidade para a comunidade acadêmica, não apenas em nível de saúde mental, mas também no que diz respeito ao processo de ensino-aprendizagem, isto é, de como a promoção de saúde poderá interferir de maneira positiva neste processo.

Por fim, quando se fala de saúde mental nos contextos universitários, não podemos esquecer de um outro agente importante e que também se encontram inserido nesses lugares, ou seja, a figura do professor. Para que a promoção de saúde nas instituições universitárias seja mais eficaz é necessária a extensão da oferta de serviços como este ao corpo docente, pois este encontra-se cotidianamente em contato com os estudantes e também possui dificuldades que podem ocasionar adoecimento e, por consequência, comprometer sua atuação tanto acadêmica, quanto relacional.

\section{Referências}

Accorsi, M. P. (2015). Atenção Psicossocial no Ambiente Universitário: Um estudo sobre a realidade dos estudantes de graduação da Universidade Federal de Santa Catarina. [Dissertação de Mestrado]. Universidade Federal de Santa Catarina.

Andrade, A. S.; Tiraboschi, G. A.; Antunes, N. A.; Viana, P. V. B. A.; Zanoto, P. A.; Curilla, R. T. (2016) Vivências Acadêmicas e Sofrimento Psíquico de Estudantes de Psicologia. Psicologia: Ciência e Profissão, 36(4), 831-846.

Araújo, C. A S. (2011). O Ambiente winnicottiano. In: E. O. Dias \& Z. Loparic (orgs.). Winnicott na Escola de São Paulo. DWW Editorial.

Biblioteca Virtual de Direitos Humano da Universidade de São Paulo. (1946) Constituição da Organização Mundial da Saúde (OMS). 
Castro, V. R. (2017). Reflexões sobre a saúde mental do estudante universitário: estudo empírico com estudantes de uma instituição pública de ensino superior. Revista Gestão em Foco, ed. 9.

Cerchiari, E. A. N.; Caetano, D.; Faccenda, O. (2005). Utilização do Serviço de Saúde Mental em uma universidade pública. Psicologia Ciência e Profissão, 25(2), 252-265.

Costa-Junior, F. M.; Maia, A. C. B. (2009). Concepções de homens hospitalizados sobre a relação entre gênero e saúde. Psicologia, Teoria e Pesquisa, 25(1), 55-63.

Coulon, A. (2017). O ofício de estudante: a entrada na vida universitária. Educação e Pesquisa, São Paulo, 43(4), 27-40.

Dias, A. C. G.; Carlotto, R. C.; Oliveira, C. T.; Teixeira, M. A. P. (2019). Dificuldades percebidas na transição para a universidade. Revista Brasileira de Orientação Profissional, 20(1), 19-30.

FONAPRACE. (2018). V Pesquisa Nacional de Perfil Socioeconômico e Cultural dos (as) Graduandos (as) das IFES, Brasília.

Gomes, R.; Moreira, M. C. N.; Nascimento, E. F.; Rebello, L. E. F. S.; Couto, M. T.; Schraiber, L. B. (2011). Os homens não vêm! Ausência e/ou invisibilidade masculina na atenção primária. Ciência \& Saúde Coletiva, 16(1), 983-992.

Mizrahi, B. G. (2008). Um contraponto ao biopoder e ao desamparo no contexto contemporâneo: reflexões winnicottianas. (Tese Doutorado. Curso de Pós-Graduação em Psicologia, Pontifícia Universidade Católica do Rio de Janeiro, Rio de Janeiro, RJ, 163 p.).

Nascimento, J. D. (2017). Mulheres nos cursos de engenharia da UFBA: um estudo sobre o acesso e desempenho. (Tese Doutorado. Programa de Pesquisa e Pós-Graduação em Educação, Universidade Federal da Bahia, Faculdade de Educação, Salvador, BA, 269 p.).

Neves, M. C. C.; Dalgalarrondo, P. (2007). Transtornos mentais auto-referidos em estudantes universitários. Jornal Brasileiro de Psiquiatria, 56 (4), 237-244.

Peres, R. S.; Santos, M. A.; Coelho, H. M. B. (2003). Atendimento psicológico a estudantes universitários: considerações acerca de uma experiência em clínica-escola. Revista Estudos de Psicologia, 20(3), 47-57. 
Rios, M. G. V.; Mascarenha, L. V. R.; Souza, K. S. S.; Olebar, D. T. C. R.; Paiva, M. C. E.; Silveira, A. O. (2019). Adoecimento e sofrimento psíquico entre universitários: estado da arte. Revista Humanidades e Inovações, 6 (8), 23-31.

Souza, S. R.; Santos, A. L.; Vieira, A. R.; Toledo, C. H.; Prado, C. M.; Moreno, C. R.; Batista, D. T.; Amaral, H. U.; Lima, J. A. C; Balsani, J. P. L.; Coelho, H, M, B.; Britto, L. O.; Bezerra, M. M.; Lopes, V. S. S.; Migliori, J. M. (2013). Pronto-atendimento psicológico a estudantes universitários: um breve estudo comparativo do perfil da clientela num intervalo de treze anos. Centro de Pesquisa e Psicologia - UNESP- Assis,SP.

Venturini, E.; Goulart, M. S. B. (2016). Universidade, solidão e saúde mental. Interfaces - Revista de Extensão da UFMG, 4 (2), 94115.

Winnicott, D. W. (1990). Natureza Humana. Rio de Janeiro: Imago.

Winnicott, D. W. (1999), O conceito de indivíduo saudável. In: D. W. Winnicott. Tudo começa em casa. São Paulo: Martins Fontes. (Original publicado em 1967).

Winnicott, D. W (1999). A imaturidade do adolescente. In: D. W. Winnicott. Tudo começa em casa. São Paulo: Martins Fontes. (Original publicado em 1968). 